\title{
Single Agents and the Set of Many-to-One Stable Matchings ${ }^{1}$
}

\author{
Ruth Martínez,* Jordi Massó, ${ }^{\dagger}$ Alejandro Neme, ${ }^{*}$ \\ and Jorge Oviedo*
}
*Instituto de Matemática Aplicada, Universidad Nacional de San Luis and CONICET, Ejército de los Andes 950, 5700 San Luis, Argentina; and †'Departament d'Economia i d'Història Econòmica and CODE, Universitat Autònoma de Barcelona, 08193 Barcelona, Spain martinez@unsl.edu.ar,jmasso@volcano.uab.es aneme@unsl.edu.ar, joviedo@unsl.edu.ar

Received March 3, 1999; revised August 3, 1999

\begin{abstract}
Some properties of the set of many-to-one stable matchings for firms that have responsive preferences and quotas are not necessarily true when firms' preferences are substitutable. In particular, we provide examples in which firms have substitutable preferences but firms and workers may be "single" in one stable matching and matched in another one. We identify a set of axioms on firms' preferences guaranteeing that the set of unmatched agents is the same under every stable matching. We also propose a weaker condition than responsiveness, called separability with quotas or $q$-separability, that together with substitutability implies this set of axioms. Journal of Economic Literature Classification Number: J41. (C) 2000 Academic Press
\end{abstract}

\section{INTRODUCTION}

Two-sided, many-to-one models have been used to study assignment problems where agents can be divided, from the very beginning, into two

${ }^{1}$ We thank José Alcade, Carmen Beviá, Flip Klijn, David Pérez-Castrillo, Howard Petith, Alvin Roth, Tayfun Sönmez, and an associate editor for helpful comments. We are especially grateful to an anonymous referee whose suggestions and comments helped to improve the paper considerably. Financial support through a grant from the Programa de Cooperación Cientifica Iberoamericana is acknowledged. The work of Jordi Massó is also partially supported by Research Grants PB96-1192 from the Dirección General de Investigación Científica y Técnica, Spanish Ministry of Education, and SGR98-62 from the Comissionat per Universitats i Recerca de la Generalitat de Catalunya. The paper was partially written while Alejandro Neme was visiting the UAB under a sabbatical fellowship from the Spanish Ministry of Education. 
disjoint subsets. One subset contains institutions, for example, firms, hospitals, colleges, sororities, orchestras, schools, and clubs. The other includes individuals, for example, workers, medical interns, students, musicians, children, and sportsmen. The fundamental question of these assignment problems consists of matching each firm on one side with a group of workers on the other side. ${ }^{2}$ Stability has been considered the main property to be satisfied by any sensible matching. A matching is called stable if all agents have acceptable partners and there is no unmatched worker-firm pair where both would prefer to be matched to each other rather than staying with their current partners. To give all blocking power to only individual agents and worker-firm pairs seems a weak requirement. Moreover, in many cases it may be the right solution concept since, to destroy an individually rational unstable matching, only a telephone call (or a couple of e-mails) is required.

The "college admissions model with substitutable preferences" is the name given by Roth and Sotomayor [9] to the most general many-to-one model with ordinal preferences. Firms are restricted to having substitutable preferences over subsets of workers; namely, all firms continue to want to employ a worker even if other workers become unavailable. ${ }^{3}$ Under this hypothesis Roth and Sotomayor [9] show that the deferred-acceptance algorithms produce either the firm-optimal stable matching or the workeroptimal stable matching, depending on whether the firms or the workers make the offers. The firm (worker)-optimal stable matching in unanimously considered by all firms (respectively, workers) to be the best among all stable matchings.

A more specific many-to-one model, called the "college admissions problem" by Gale and Shapley [1], supposes that firms (colleges) have a maximum number of positions to be filled (their quota) and that each firm (college), given its ranking of individual workers (students), orders subsets of workers in a responsive manner; namely, for any two subsets that differ in only one student, a college prefers the subset containing the most-preferred student. In this model the set of stable matchings satisfies the following additional properties: (1) There is a polarization of interests between the two sides of the market along the set of stable matchings. (2) The set of unmatched agents is the same under every stable matching. (3) The number of workers assigned to a firm through stable matchings is the same. (4) If a firm does

\footnotetext{
${ }^{2}$ We will follow the convention of generically referring to institutions as firms and to individuals as workers. See Roth and Sotomayor [9] for an illuminating and comprehensive survey of this literature as well as an exhaustive bibliography.

${ }^{3}$ Kelso and Crawford [4] were the first to use this property in a more general model with money. They proved the existence of a stable matching and of a firm-optimal stable matching (all firms agree it is the best stable matching).
} 
not complete its quota under some stable matching, then it gets the same set of workers at any stable matching. ${ }^{4}$

The purpose of this note is twofold. On the one hand, its negative side is that we exhibit examples of firms having substitutable preferences in which properties (1) to (4) are violated. On the other hand, its positive side is that we propose a set of axioms on firms' preferences (opposite optimality, acceptability, and desirability) under which properties (2), (3), and (4) hold. Moreover, we identify a weaker condition than $q$-responsiveness, called separability with quota, or $q$-separability, that together with substitutability implies the set of axioms. We also show that these restricted preferences do not guarantee that property (1) holds (see Example 2). A firm is said to have separable preferences over all subsets of workers if its partition between acceptable and unacceptable workers has the property that only adding acceptable workers makes any given subset of workers a better one. However, in many applications, such as entry-level professional labor markets, separability alone does not seem very reasonable because firms usually have a much smaller number of openings (their quota) than there are "good" workers looking for a job. In those cases it seems reasonable to restrict firm preferences in such a way that the separability condition operates only up to their quota, considering unacceptable all subset with higher cardinality. Moreover, while responsiveness seems the relevant property for extending an ordered list of individual students to preferences on all subsets of students, it is too restrictive to capture some degree of complementarity among workers, which can be very natural in other settings. The $q$-separability condition permits greater flexibility in going from orders on individuals to orders on subsets. For instance, candidates for a job can be grouped together by areas of specialization. A firm with quota 2 may consider as the best subset of workers not the set consisting of the first two candidates on the individual ranking (who may both have the same specialization), but rather the subset composed of the first and fourth candidates in the individual ranking (i.e., the first in each area of specialization).

In the next section we present the notation and definitions. Section 3 contains the results and the main examples.

\section{NOTATION AND DEFINITIONS}

There are two disjoint sets of agents, the set of $n$ firms $\mathscr{F}$ and the set of $m$ workers $\mathscr{W}$. Each firm $F \in \mathscr{F}$ has a strict, transitive, and complete

${ }^{4}$ Property (1) is a consequence of the decomposition lemma proved by Gale and Sotomayor $[2,3]$. Properties (2) and (3) were proved independently by Gale and Sotomayor [2, 3] and Roth [5]. Property (4) was proved by Roth [7]. 
preference relation $P(F)$ over $2^{\mathscr{W}}$, and each worker $w \in \mathscr{W}$ has a strict, transitive, and complete preference relation $P(w)$ over $\mathscr{F} \cup\{\varnothing\}$. Preference profiles are $(n+m)$-tuples of preference relations and they are represented by $P=\left(P\left(F_{1}\right), \ldots, P\left(F_{n}\right) ; P\left(w_{1}\right), \ldots, P\left(w_{m}\right)\right)$. Given a preference relation of a firm $P(F)$ the subsets of workers preferred to the empty set by $F$ are called acceptable. Similarly, given a preference relation of a worker $P(w)$ the firms preferred by $w$ to the empty set are called acceptable. To express preference relations in a concise manner, and since only acceptable partners will matter, we will represent preference relations as lists of acceptable partners. We will denote by $\mathscr{P}$ a generic subset of preference profiles.

The assignment problem consists of matching workers with firms, keeping the bilateral nature of their relationship and allowing for the possibility that both firms and workers may remain unmatched. Formally,

Definition 1. A matching $\mu$ is a mapping from the set $\mathscr{F} \cup \mathscr{W}$ into the set of all subsets of $\mathscr{F} \cup \mathscr{W}$ such that for all $w \in \mathscr{W}$ and $F \in \mathscr{F}$ :

1. Either $|\mu(w)|=1$ and $\mu(w) \subseteq \mathscr{F}$ or else $\mu(w)=\varnothing$.

2. $\mu(F) \in 2^{\mathscr{W}}$.

3. $\mu(w)=F$ if and only if $w \in \mu(F)$.

We say that $w$ and $F$ are single in a matching $\mu$ if $\mu(w)=\varnothing$ and $\mu(F)=\varnothing$. Otherwise, they are matched. A matching $\mu$ is said to be one-to-one if firms can hire at most one worker; namely, condition 2 is replaced by either $|\mu(F)|=1$ and $\mu(F) \subseteq \mathscr{W}$ or else $\mu(F)=\varnothing$. The model in which all matchings are oneto-one is also known in the literature as the marriage model. We will follow the widespread notation where

$$
\mu=\left(\begin{array}{cccc}
F_{1} & F_{2} & F_{3} & \varnothing \\
\left\{w_{3}, w_{4}\right\} & \left\{w_{1}\right\} & \varnothing & \left\{w_{2}\right\}
\end{array}\right)
$$

represents the obvious matching between $\mathscr{F}=\left\{F_{1}, F_{2}, F_{3}\right\}$ and $\mathscr{W}=\left\{w_{1}\right.$, $\left.w_{2}, w_{3}, w_{4}\right\}$.

Given a set of workers $S \subseteq \mathscr{W}$, let $\operatorname{Ch}(S, P(F))$ denote $F$ 's most preferred subset of $S$ according to its preference ordering $P(F)$. A matching $\mu$ is blocked by a worker $w$ if $\varnothing P(w) \mu(w)$. Similarly, $\mu$ is blocked by a firm $F$ if $\mu(F) \neq \mathrm{Ch}(\mu(F), P(F))$. We say that a matching is individually rational if it is not blocked by any individual agent. A matching $\mu$ is blocked by a worker-firm pair $(w, F)$ if $F P(w) \mu(w)$ and $w \in \mathrm{Ch}(\mu(F) \cup\{w\}, P(F))$.

Definition 2. A matching $\mu$ is stable if it is not blocked by any individual agent or any firm-worker pair. 
Given a preference profile $P$, denote the set of stable matchings by $S(P)$. It is easy to construct examples of preference profiles $P$ with the property that the set $S(P)$ is empty. This is why the literature has focused on the restriction that workers are regarded as substitutes.

Definition 3. A firm $F$ 's preference ordering $P(F)$ satisfies substitutability if for any set $S$ containing workers $w$ and $\bar{w}(w \neq \bar{w})$, if $w \in \operatorname{Ch}(S, P(F))$ then $w \in \operatorname{Ch}(S \backslash\{\bar{w}\}, P(F))$.

A preference profile $P$ is substitutable if for each firm $F$, the preference ordering $P(F)$ satisfies substitutability. The literature has also studied subsets of preferences where firms (workers) unanimously agree that a matching $\mu_{\mathscr{F}}\left(\mu_{\mathscr{W}}\right)$ is the best stable matching. That is why $\mu_{\mathscr{F}}$ and $\mu_{\mathscr{W}}$ are called, respectively, the firm-optimal stable matching and the worker-optimal stable matching. ${ }^{5}$ Moreover, there is an opposition of interests on these two optimal stable matchings. We state both properties as an axiom on sets of preferences profiles $\mathscr{P}$.

(OO) The set $\mathscr{P}$ satisfies Opposite Optimality if for all $P \in \mathscr{P}$ there exists $\mu_{\mathscr{F}}, \mu_{\mathscr{W}} \in S(P)$ such that for all $\mu \in S(P)$ and all $F$ and $w: \mu_{\mathscr{F}} R(F)$ $\mu R(F) \mu_{\mathscr{W}}$ and $\mu_{\mathscr{W}} R(w) \mu R(w) \mu_{\mathscr{F}}$.

Remark 1. The set of substitutable preferences satisfies opposite optimality.

We will also concentrate on subsets of preferences satisfying the axiom that, in a stable matching, firms only hire individually acceptable workers.

(A) The set $\mathscr{P}$ satisfies Acceptability if for all $P \in \mathscr{P}$ and all $\mu \in S(P)$ : $w \in \mu(F)$ implies $w P(F) \varnothing$.

We will assume that each firm $F$ has a maximum number of positions to be filled: its quota $q_{F}$. This limitation may arise from, for example, technological, legal, or budgetary reasons. We will denote by $q=\left(q_{F}\right)_{F \in \mathscr{F}}$ the list of quotas and we will focus on the axiom that if a firm does not fill its quota it is willing to hire an acceptable worker. Formally,

(D) The set $\mathscr{P}$ satisfies $q$-desirability if for all $P \in \mathscr{P}$, all $\mu \in S(P)$, and all $F \in \mathscr{F}:|\mu(F)|<q_{F}$ and $w P(F) \varnothing$ imply $w \in \operatorname{Ch}(\mu(F) \cup\{w\}, P(F))$.

\footnotetext{
${ }^{5}$ We are following the convention of extending preferences from the original sets $\left(2^{\mathscr{W}}\right.$ and $\mathscr{F} \cup\{\varnothing\})$ to the set of matchings. However, we now have to consider weak orderings since the matchings $\mu$ and $\mu^{\prime}$ may associate the same partner with an individual. These orderings will be denoted by $R(F)$ and $R(w)$.
} 
We now define the set of $q$-separable and $q$-responsive preferences. A firm $F$ has $q$-separable preferences if the division between good workers $(w P(F) \varnothing)$ and bad workers $(\varnothing P(F) w)$ guides, up to its quota, the ordering of subsets in the sense that adding a good workers leads to a better set, while adding a bad worker leads to a worse set. Formally,

Definition 4. A firm $F$ 's preference ordering $P(F)$ is $q_{F}$-separable if: (a) for all $S \subsetneq \mathscr{W}$ such that $|S|<q_{F}$ and $w \notin S$ we have that $(S \cup\{w\}) P(F) S$ if and only if $w P(F) \varnothing$, and (b) $\varnothing P(F) S$ for all $S$ such that $|S|>q_{F}{ }^{6}$

Remark 2. The set of $q$-separable preferences satisfies acceptability and $q$-desirability.

Following Roth and Sotomayor [9], a firm $F$ 's preference ordering $P(F)$ (over all subsets of workers) is said to be $q_{F}$-responsive (to its ordering over individual workers) if it is $q_{F}$-separable and for any two sets of workers that differ in only one worker, $F$ prefers the subset containing the mostpreferred worker. Formally,

Definition 5. A firm $F$ 's preference ordering $P(F)$ over sets of workers is $q_{F}$-responsive if it is $q_{F}$-separable and for all $S, w^{\prime} \in S$, and $w \notin S$ we have that $\left(S \backslash\left\{w^{\prime}\right\} \cup\{w\}\right) P(F) S$ if and only if $w P(F) w^{\prime}$.

A preference profile $P$ is $q$-separable if each $P(F)$ is $q_{F}$-separable. Similarly, $P$ is $q$-responsive if each $P(F)$ is $q_{F}$-responsive. In principle, we may have firms with different quotas. The case where all firms have 1-separable preferences is equivalent, from the point of view of stability, to the one-toone model. Hence, our set-up includes the marriage model as a particular case.

The following ordering over $2^{\mathscr{W}}$, where $\mathscr{W}=\left\{w_{1}, w_{2}, w_{3}, w_{4}\right\}$,

$$
\begin{aligned}
P(F)= & \left\{w_{1}, w_{2}\right\},\left\{w_{3}, w_{4}\right\},\left\{w_{1}, w_{3}\right\},\left\{w_{1}, w_{4}\right\},\left\{w_{2}, w_{3}\right\},\left\{w_{2}, w_{4}\right\}, \\
& \left\{w_{1}\right\},\left\{w_{2}\right\},\left\{w_{3}\right\},\left\{w_{4}\right\}
\end{aligned}
$$

illustrates the fact that $q$-separability does not imply substitutability. To see this, notice that $P(F)$ is 2-separable but it is not substitutable since $w_{1} \in \mathrm{Ch}\left(\left\{w_{1}, w_{2}, w_{3}, w_{4}\right\}, P(F)\right)$, but $w_{1} \notin \mathrm{Ch}\left(\left\{w_{1}, w_{3}, w_{4}\right\}, P(F)\right)$. However,

\footnotetext{
${ }^{6}$ For the purpose of studying the set of stable matchings, condition (b) in this definition could be replaced by the following condition: $|\mathrm{Ch}(S, P(F))| \leqslant q_{F}$ for all $S$ such that $|S|>q_{F}$. We choose condition (b) since it is simpler. Sönmez [10] uses an alternative approach that consists of deleting condition (b) in the definition but then requiring in the definition of a matching that $|\mu(F)| \leqslant q_{F}$ for all $F \in \mathscr{F}$.
} 
it is easy to see that all $(m-1)$-separable as well as all $q_{F}$-responsive preferences are substitutable. As a consequence of this latter inclusion we have that $S(P) \neq \varnothing$ if $P$ is $q$-responsive. The ordering

$$
P\left(F^{\prime}\right)=\left\{w_{1}, w_{3}\right\},\left\{w_{1}, w_{2}\right\},\left\{w_{2}, w_{3}\right\},\left\{w_{1}\right\},\left\{w_{2}\right\},\left\{w_{3}\right\}
$$

illustrates the fact that the set of $q$-responsive preferences is a proper subset of the set of $q$-separable and substitutable preferences.

The following example shows that even if all firms have $q$-separable preferences the set of stable matchings may be empty.

EXAMPLE 1. Let $\mathscr{F}=\left\{F_{1}, F_{2}\right\}$ and $\mathscr{W}=\left\{w_{1}, w_{2}, w_{3}, w_{4}\right\}$ be the two sets of agents with a profile of preferences $P$ defined by

$$
\begin{aligned}
P\left(F_{1}\right)= & \left\{w_{3}, w_{4}\right\},\left\{w_{2}, w_{4}\right\},\left\{w_{1}, w_{2}\right\},\left\{w_{1}, w_{3}\right\}, \\
& \left\{w_{2}, w_{3}\right\},\left\{w_{1}, w_{4}\right\},\left\{w_{1}\right\},\left\{w_{2}\right\},\left\{w_{3}\right\},\left\{w_{4}\right\}, \\
P\left(F_{2}\right)= & \left\{w_{3}\right\},\left\{w_{4}\right\}, \\
P\left(w_{1}\right)= & F_{1}, \\
P\left(w_{2}\right)= & F_{1}, \\
P\left(w_{3}\right)= & F_{1}, F_{2}, \quad \text { and } \\
P\left(w_{4}\right)= & F_{2}, F_{1} .
\end{aligned}
$$

Notice that $P$ is $(2,1)$-separable. However, it is a matter of verification that $S(P)=\varnothing$.

\section{RESULTS AND EXAMPLES}

In the marriage model the decomposition lemma stays that both sides of the market are in conflict on the set of stable matchings in the sense that the partners (through any stable matching $\mu_{1}$ ) of the subset of agents of one side of the market that consider $\mu_{1}$ to be at least as good as the stable matching $\mu_{2}$, have to consider $\mu_{2}$ to be at least as good as $\mu_{1}$. In particular (and we referred to it in the Introduction as Property (1)), if all agents of one side of the market consider the stable matching $\mu_{1}$ to be at least as good as the stable matching $\mu_{2}$ then all agents of the other side have to consider $\mu_{2}$ to be at least as good as $\mu_{1}$. For the college admissions model 
with $q$-responsive preferences the decomposition lemma can be obtained by using the following result of Roth and Sotomayor [8]: For any given pair of stable matchings $\mu_{1}$ and $\mu_{2}$, if firm $F$ prefers $\mu_{1}(F)$ to $\mu_{2}(F)$ then it prefers every worker in $\mu_{1}(F)$ to any worker in $\mu_{2}(F) \backslash \mu_{1}(F)$. This result also implies that the set of stable matchings depends only on how firms order individual workers and not on their specific responsive extensions. Roth [6] gives an example where the decomposition lemma is not true in a many-to-one model with money and substitutable preferences. Example 2 below shows that the decomposition lemma does not hold in our more restricted framework of ordinal, $q$-separable, and substitutable preferences.

EXAmple 2. Let $\mathscr{F}=\left\{F_{1}, F_{2}\right\}$ and $\mathscr{W}=\left\{w_{1}, w_{2}, w_{3}, w_{4}\right\}$ be the two sets of agents with $(2,2)$-separable and substitutable profile of preferences $P$ defined by

$$
\begin{aligned}
P\left(F_{1}\right)= & \left\{w_{1}, w_{2}\right\},\left\{w_{1}, w_{3}\right\},\left\{w_{2}, w_{4}\right\},\left\{w_{3}, w_{4}\right\}, \\
& \left\{w_{1}, w_{4}\right\},\left\{w_{2}, w_{3}\right\},\left\{w_{1}\right\},\left\{w_{2}\right\},\left\{w_{3}\right\},\left\{w_{4}\right\}, \\
P\left(F_{2}\right)= & \left\{w_{3}, w_{4}\right\},\left\{w_{2}, w_{4}\right\},\left\{w_{1}, w_{3}\right\},\left\{w_{1}, w_{2}\right\}, \\
& \left\{w_{1}, w_{4}\right\},\left\{w_{2}, w_{3}\right\},\left\{w_{1}\right\},\left\{w_{2}\right\},\left\{w_{3}\right\},\left\{w_{4}\right\}, \\
P\left(w_{1}\right)= & F_{2}, F_{1}, \\
P\left(w_{2}\right)= & F_{2}, F_{1}, \\
P\left(w_{3}\right)= & F_{1}, F_{2}, \\
P\left(w_{4}\right)= & F_{1}, F_{2} .
\end{aligned}
$$

The set of stable matchings consists of the following four matchings:

$$
\begin{aligned}
& \mu_{\mathscr{F}}=\left(\begin{array}{cc}
F_{1} & F_{2} \\
\left\{w_{1}, w_{2}\right\} & \left\{w_{3}, w_{4}\right\}
\end{array}\right), \quad \mu_{1}=\left(\begin{array}{cc}
F_{1} & F_{2} \\
\left\{w_{1}, w_{3}\right\} & \left\{w_{2}, w_{4}\right\}
\end{array}\right), \\
& \mu_{2}=\left(\begin{array}{cc}
F_{1} & F_{2} \\
\left\{w_{2}, w_{4}\right\} & \left\{w_{1}, w_{3}\right\}
\end{array}\right),
\end{aligned}
$$

and

$$
\mu_{\mathscr{W}}=\left(\begin{array}{cc}
F_{1} & F_{2} \\
\left\{w_{3}, w_{4}\right\} & \left\{w_{1}, w_{2}\right\}
\end{array}\right) .
$$


Notice that $\mu_{\mathscr{F}} P(F) \mu_{1} P(F) \mu_{2} P(F) \mu_{\mathscr{W}}$ for all $F$ and

$$
\begin{aligned}
& \mu_{\mathscr{W}} R\left(w_{1}\right) \mu_{2} P\left(w_{1}\right) \mu_{1} R\left(w_{1}\right) \mu_{\mathscr{F}}, \\
& \mu_{\mathscr{W}} R\left(w_{2}\right) \mu_{1} P\left(w_{2}\right) \mu_{2} R\left(w_{2}\right) \mu_{\mathscr{F}}, \\
& \mu_{\mathscr{W}} R\left(w_{3}\right) \mu_{1} P\left(w_{3}\right) \mu_{2} R\left(w_{3}\right) \mu_{\mathscr{F}},
\end{aligned}
$$

and

$$
\mu_{\mathscr{W}} R\left(w_{4}\right) \mu_{2} P\left(w_{4}\right) \mu_{1} R\left(w_{4}\right) \mu_{\mathscr{F}} .
$$

Therefore, $\mu_{1}$ is strictly preferred to $\mu_{2}$ by $F_{1}, F_{2}$ and $w_{2}$.

We now establish that under axioms (OO), (A), and (D) (and therefore, under $q$-separable and substitutable preferences) properties (2), (3), and (4) hold. These are important properties; for instance, if property (2) does not hold, a "single" agent would be able to argue that he was badly treated by a particular stable matching. Remember that they also hold in both the marriage and the college admissions models. This is in spite of the fact that their proofs in these models, according to Roth and Sotomayor [9], are "a simple consequence of the decomposition lemma," which does not hold in our setting. Moreover, the structure of the set of stable matchings coincide in the marriage and the college admissions models. This is because one can identify each firm $F$ with $q_{F}$ identical firms and any many-to-one matching $\mu$ with the one-to-one matching $\bar{\mu}$ where each $w \in \mu(F)$ is matched through $\bar{\mu}$ with one of the $q_{F}$ replica of $F$. However, as soon as preferences are not $q$-responsive, the properties of the set of stable matchings have to be proven directly without relying on the properties of the marriage model.

Proposition 1. Assume $\mathscr{P}$ satisfies axioms (OO), (A), and (D) and let $P \in \mathscr{P}$. If $w$ is single in $\mu \in S(P)$, then $w$ is single in any $\mu^{\prime} \in S(P)$.

Proof. Suppose the contrary; that is, there exist $\bar{w}, \hat{F}$, and $\mu, \mu^{\prime} \in S(P)$ such that $\mu(\bar{w})=\varnothing$ and $\mu^{\prime}(\bar{w})=\hat{F}$. By $(\mathrm{OO})$, there exist matchings $\mu_{\mathscr{F}}$ and $\mu_{\mathscr{W}}$ that are the worst and the best stable matchings, respectively, for all workers. Therefore, we can also find a firm $\bar{F}$ such that $\bar{w} \in \mu_{\mathscr{W}}(\bar{F})$ and $\bar{w} \notin \bigcup_{F} \mu_{\mathscr{F}}(F)$. We will distinguish between two cases:

Case 1. $\bigcup_{F} \mu_{\mathscr{F}}(F) \subseteq \bigcup_{F} \mu_{\mathscr{W}}(F)$. In this case

$$
\sum_{F}\left|\mu_{\mathscr{F}}(F)\right|<\sum_{F}\left|\mu_{\mathscr{W}}(F)\right| \leqslant \sum_{F} q_{F},
$$

where the strict inequality follows because $\bar{w} \notin \bigcup_{F} \mu_{\mathscr{F}}(F)$. Then $\left|\mu_{\mathscr{F}}(\widetilde{F})\right|<$ $q_{\tilde{F}}$ for at least one $\widetilde{F}$. Denote by $\tilde{\mathscr{F}}$ the set of all such firms. We claim that 
there exists $F_{0} \in \tilde{\mathscr{F}}$ such that $\mu_{\mathscr{W}}\left(F_{0}\right) \backslash \mu_{\mathscr{F}}\left(F_{0}\right) \neq \varnothing$, because otherwise, $\mu_{\mathscr{W}}(F) \subseteq \mu_{\mathscr{F}}(F)$ for all $F \in \tilde{\mathscr{F}}$ would imply

$$
\begin{aligned}
\sum_{F}\left|\mu_{\mathscr{W}}(F)\right| & =\left|\bigcup_{F} \mu_{\mathscr{W}}(F)\right|=\left|\bigcup_{F \in \tilde{\mathscr{F}}} \mu_{\mathscr{W}}(F)\right|+\left|\bigcup_{F \notin \tilde{\mathscr{F}}} \mu_{\mathscr{W}}(F)\right| \\
& \leqslant\left|\bigcup_{F \in \tilde{\mathscr{F}}} \mu_{\mathscr{F}}(F)\right|+\left|\bigcup_{F \notin \tilde{\mathscr{F}}} \mu_{\mathscr{W}}(F)\right| \\
& \leqslant\left|\bigcup_{F \in \tilde{\mathscr{F}}} \mu_{\mathscr{F}}(F)\right|+\left|\bigcup_{F \notin \tilde{\mathscr{F}}} \mu_{\mathscr{F}}(F)\right| \\
& =\left|\bigcup_{F} \mu_{\mathscr{F}}(F)\right|=\sum_{F}\left|\mu_{\mathscr{F}}(F)\right|,
\end{aligned}
$$

which contradicts (1). Let $w_{0} \in \mu_{\mathscr{W}}\left(F_{0}\right) \backslash \mu_{\mathscr{F}}\left(F_{0}\right)$. Then, the pair $\left(w_{0}, F_{0}\right)$ blocks $\mu_{\mathscr{F}}$, since we have that $w_{0} \notin \mu_{\mathscr{F}}\left(F_{0}\right), F_{0}=\mu_{\mathscr{W}}\left(w_{0}\right) P\left(w_{0}\right) \mu_{\mathscr{F}}\left(w_{0}\right)$, and

$$
w_{0} \in \operatorname{Ch}\left(\mu_{\mathscr{F}}\left(F_{0}\right) \cup\left\{w_{0}\right\}, P\left(F_{0}\right)\right) .
$$

Condition (2) holds because $\left|\mu_{\mathscr{F}}\left(F_{0}\right)\right|<q_{F_{0}}$ and $w_{0} \in \mu_{\mathscr{W}}\left(F_{0}\right)$ imply, by (A) and (D), that $w_{0} P\left(F_{0}\right) \varnothing$. Therefore, Case 1 is false.

Case 2. $\bigcup_{F} \mu_{\mathscr{F}}(F) \nsubseteq \bigcup_{F} \mu_{\mathscr{W}}(F)$. In this case, there exists a worker $\tilde{w} \in \bigcup_{F} \mu_{\mathscr{F}}(F) \backslash \bigcup_{F} \mu_{\mathscr{W}}(F)$. Hence, we can find $\tilde{F}$ such that $\tilde{w} \in \mu_{\mathscr{F}}(\tilde{F})$ while $\tilde{w} \notin \bigcup_{F} \mu_{\mathscr{W}}(F)$. But this says that by $(\mathrm{OO}), \mu_{\mathscr{W}}(\tilde{w})=\varnothing P(\tilde{w}) \mu_{\mathscr{F}}(\tilde{w})=\tilde{F}$, which contradicts that $\mu_{\mathscr{F}}$ is individually rational for $\tilde{w}$.

Proposition 2 below states that properties (3) and (4) also hold under our set of axioms (remember that they hold for the college admissions model).

Proposition 2. Assume $\mathscr{P}$ satisfies axioms (OO), (A), and (D). Then, for all $P \in \mathscr{P}$, all pairs $\mu, \mu^{\prime} \in S(P)$, and all $F \in \mathscr{F}$ :

(a) $|\mu(F)|=\left|\mu^{\prime}(F)\right|$.

(b) If $|\mu(F)|<q_{F}$ then $\mu(F)=\mu^{\prime}(F)$.

Proof. To prove (a) we will show that if $\mu \in S(P)$ then $|\mu(F)|=\left|\mu_{\mathscr{W}}(F)\right|$ for all $F \in \mathscr{F}$. Assume the contrary; that is, suppose there exist $\mu \in S(P)$ and $F \in \mathscr{F}$ such that $|\mu(F)| \neq\left|\mu_{\mathscr{W}}(F)\right|$.

Case 1. Assume $\left|\mu_{\mathscr{W}}(F)\right|>|\mu(F)|$ holds. This means that there exists $w \in \mu_{\mathscr{W}}(F) \backslash \mu(F)$. Therefore, 
By $(\mathrm{OO})$ we have that

$$
F=\mu_{\mathscr{W}}(w) P(w) \mu(w)
$$

By (A) and $w \in \mu_{\mathscr{W}}(F)$ we have that $w P(F) \varnothing$, which implies, by (D), that

$$
w \in \mathrm{Ch}(\mu(F) \cup\{w\}, P(F))
$$

since $|\mu(F)|<q_{F}$. Therefore, conditions (3), (4), and (5) imply that $\mu$ is not stable since $(w, F)$ blocks it.

Case 2. Assume $\left|\mu_{\mathscr{W}}(F)\right|<|\mu(F)|$ holds. We claim that we can find $\hat{F} \in \mathscr{F}$ such that $|\mu(\hat{F})|<\left|\mu_{\mathscr{W}}(\hat{F})\right|$; otherwise the number of workers matched at $\mu$ would be greater than the number of workers matched at $\mu_{\mathscr{W}}$, contradicting Proposition 1. Applying Case 1 to $\hat{F}$ we conclude that $\mu$ is not stable.

To prove (b) suppose that $\mu \in S(P)$ and $|\mu(F)|<q_{F}$ for some $F \in \mathscr{F}$. By (a) we have that $\left|\mu_{\mathscr{W}}(F)\right|=|\mu(F)|<q_{F}$ holds. It is sufficient to show that $\mu(F)=\mu_{\mathscr{W}}(F)$. To get a contradiction let $w \in \mu_{\mathscr{W}}(F) \backslash \mu(F)$ and, by Proposition 1 , let $F^{\prime} \neq F$ be such that

$$
w \in \mu\left(F^{\prime}\right) .
$$

Using (A) we obtain that $w P(F) \varnothing$ and (OO) imply that

$$
\mu_{\mathscr{W}}(w)=F P(w) F^{\prime}=\mu(w) .
$$

Therefore, $w P(F) \varnothing$ and $|\mu(F)|<q_{F}$ imply, by (D), that

$$
w \in \mathrm{Ch}(\mu(F) \cup\{w\}, P(F)) .
$$

Conditions (6), (7), and (8) imply that ( $w, F)$ blocks $\mu$.

Since Proposition 2 implies that the set of unmatched firms is the same in all stable matchings, we can state the following corollary.

COROLlary 3. Assume firms have q-separable and substitutable preferences. Then, the set of unmatched agents is the same under every stable matching.

Examples 3, 4, 5, and 6 below show that the statement of Corollary 3 is false without either $q$-separability or substitutability.

ExAmple 3. Let $\mathscr{F}=\left\{F_{1}, F_{2}\right\}$ and $\mathscr{W}=\left\{w_{1}, w_{2}, w_{3}, w_{4}\right\}$ be the two sets of agents with a substitutable profile of preferences $P$ defined by 


$$
\begin{aligned}
P\left(F_{1}\right)= & \left\{w_{1}, w_{2}\right\},\left\{w_{1}, w_{3}\right\},\left\{w_{2}, w_{4}\right\},\left\{w_{3}, w_{4}\right\}, \\
& \left\{w_{1}\right\},\left\{w_{2}\right\},\left\{w_{3}\right\},\left\{w_{4}\right\}, \\
P\left(F_{2}\right)= & \left\{w_{3}\right\},\left\{w_{1}, w_{2}\right\},\left\{w_{1}\right\},\left\{w_{2}\right\},\left\{w_{4}\right\}, \\
P\left(w_{1}\right)= & F_{2}, F_{1}, \\
P\left(w_{2}\right)= & F_{2}, F_{1}, \\
P\left(w_{3}\right)= & F_{1}, F_{2}, \quad \text { and } \\
P\left(w_{4}\right)= & F_{1}, F_{2} .
\end{aligned}
$$

The ordering $P\left(F_{2}\right)$ is not $q_{F_{2}}$-separable and any $\mathscr{P}$ containing $P$ would violate axiom (D) for any $q_{F_{2}} \geqslant 1$. The two optimal stable matchings

$$
\mu_{\mathscr{F}}=\left(\begin{array}{ccc}
F_{1} & F_{2} & \varnothing \\
\left\{w_{1}, w_{2}\right\} & \left\{w_{3}\right\} & \left\{w_{4}\right\}
\end{array}\right)
$$

and

$$
\mu_{\mathscr{W}}=\left(\begin{array}{cc}
F_{1} & F_{2} \\
\left\{w_{3}, w_{4}\right\} & \left\{w_{1}, w_{2}\right\}
\end{array}\right)
$$

have the property that $\mu_{\mathscr{F}}\left(w_{4}\right)=\varnothing$ and $\mu_{\mathscr{W}}\left(w_{4}\right)=F_{1}$.

EXAMPLE 4. Let $\mathscr{F}=\left\{F_{1}, F_{2}\right\}$ and $\mathscr{W}=\left\{w_{1}, w_{2}, w_{3}, w_{4}, w_{5}\right\}$ be the two sets of agents with the $(2,2)$-separable profile of preferences $P$ defined by

$$
\begin{aligned}
P\left(F_{1}\right)= & \left\{w_{1}, w_{2}\right\},\left\{w_{3}, w_{4}\right\},\left\{w_{1}, w_{3}\right\},\left\{w_{1}, w_{4}\right\}, \\
& \left\{w_{2}, w_{3}\right\},\left\{w_{2}, w_{4}\right\},\left\{w_{1}\right\},\left\{w_{2}\right\},\left\{w_{3}\right\},\left\{w_{4}\right\}, \\
P\left(F_{2}\right)= & \left\{w_{3}, w_{5}\right\},\left\{w_{1}, w_{2}\right\},\left\{w_{1}, w_{3}\right\},\left\{w_{1}, w_{5}\right\}, \\
& \left\{w_{2}, w_{3}\right\},\left\{w_{2}, w_{5}\right\},\left\{w_{1}\right\},\left\{w_{2}\right\},\left\{w_{3}\right\},\left\{w_{5}\right\}, \\
P\left(w_{1}\right)= & F_{2}, F_{1}, \\
P\left(w_{2}\right)= & F_{2}, F_{1}, \\
P\left(w_{3}\right)= & F_{1}, F_{2}, \\
P\left(w_{4}\right)= & F_{1}, F_{2}, \quad \text { and } \\
P\left(w_{5}\right)= & F_{2} .
\end{aligned}
$$

Notice that $P\left(F_{1}\right)$ is not substitutable and the two stable matchings

$$
\mu_{1}=\left(\begin{array}{ccc}
F_{1} & F_{2} & \varnothing \\
\left\{w_{1}, w_{2}\right\} & \left\{w_{3}, w_{5}\right\} & \left\{w_{4}\right\}
\end{array}\right)
$$


and

$$
\mu_{2}=\left(\begin{array}{ccc}
F_{1} & F_{2} & \varnothing \\
\left\{w_{3}, w_{4}\right\} & \left\{w_{1}, w_{2}\right\} & \left\{w_{5}\right\}
\end{array}\right)
$$

have different single workers. Any $\mathscr{P}$ containing $P$ would violate axiom (OO) since $\mu_{\mathscr{W}}$ does not exist and $\mu_{1}=\mu_{\mathscr{F}}$ is not the worst stable matching for $w_{5}$.

EXAmple 5. Let $\mathscr{F}=\left\{F_{1}, F_{2}, F_{3}\right\}$ and $\mathscr{W}=\left\{w_{1}, w_{2}, w_{3}, w_{4}\right\}$ be the two sets of agents with a substitutable profile of preferences $P$ defined by

$$
\begin{aligned}
P\left(F_{1}\right)= & \left\{w_{1}, w_{2}\right\},\left\{w_{1}, w_{3}\right\},\left\{w_{1}, w_{4}\right\},\left\{w_{2}, w_{3}\right\}, \\
& \left\{w_{2}, w_{4}\right\},\left\{w_{3}, w_{4}\right\},\left\{w_{1}\right\},\left\{w_{2}\right\},\left\{w_{3}\right\},\left\{w_{4}\right\}, \\
P\left(F_{2}\right)= & \left\{w_{3}\right\},\left\{w_{1}, w_{3}\right\},\left\{w_{2}, w_{3}\right\},\left\{w_{1}, w_{2}\right\},\left\{w_{1}\right\},\left\{w_{2}\right\}, \\
P\left(F_{3}\right)= & \left\{w_{4}\right\}, \\
P\left(w_{1}\right)= & F_{2}, F_{1}, \\
P\left(w_{2}\right)= & F_{2}, F_{1}, \\
P\left(w_{3}\right)= & F_{1}, F_{2}, \\
P\left(w_{4}\right)= & F_{1}, F_{3} .
\end{aligned}
$$

The ordering $P\left(F_{2}\right)$ is not $q_{F_{2}}$-separable and any $\mathscr{P}$ containing $P$ would violate axiom (D) for any $q_{F_{2}} \geqslant 1$. The two optimal stable matchings

$$
\mu_{\mathscr{F}}=\left(\begin{array}{ccc}
F_{1} & F_{2} & F_{3} \\
\left\{w_{1}, w_{2}\right\} & \left\{w_{3}\right\} & \left\{w_{4}\right\}
\end{array}\right)
$$

and

$$
\mu_{\mathscr{W}}=\left(\begin{array}{ccc}
F_{1} & F_{2} & F_{3} \\
\left\{w_{3}, w_{4}\right\} & \left\{w_{1}, w_{2}\right\} & \varnothing
\end{array}\right)
$$

have the property that $\mu_{\mathscr{F}}\left(F_{3}\right) \neq \varnothing$ and $\mu_{\mathscr{W}}\left(F_{3}\right)=\varnothing$.

EXAMPLE 6. Let $\mathscr{F}=\left\{F_{1}, F_{2}, F_{3}\right\}$ and $\mathscr{W}=\left\{w_{1}, w_{2}, w_{3}, w_{4}, w_{5}\right\}$ be the two sets of agents with a $(2,2,2)$-separable profile of preferences $P$ defined by 


$$
\begin{aligned}
P\left(F_{1}\right)= & \left\{w_{1}, w_{2}\right\},\left\{w_{3}, w_{5}\right\},\left\{w_{2}, w_{5}\right\},\left\{w_{1}, w_{5}\right\}, \\
& \left\{w_{1}, w_{3}\right\},\left\{w_{2}, w_{3}\right\},\left\{w_{1}\right\},\left\{w_{2}\right\},\left\{w_{3}\right\},\left\{w_{5}\right\}, \\
P\left(F_{2}\right)= & \left\{w_{3}, w_{4}\right\},\left\{w_{1}, w_{2}\right\},\left\{w_{2}, w_{4}\right\},\left\{w_{1}, w_{4}\right\},\left\{w_{1}, w_{3}\right\}, \\
& \left\{w_{2}, w_{3}\right\},\left\{w_{3}\right\},\left\{w_{1}, w_{2}\right\},\left\{w_{1}\right\},\left\{w_{2}\right\},\left\{w_{3}\right\},\left\{w_{4}\right\}, \\
P\left(F_{3}\right)= & \left\{w_{5}\right\}, \\
P\left(w_{1}\right)= & F_{2}, F_{1}, \\
P\left(w_{2}\right)= & F_{2}, F_{1}, \\
P\left(w_{3}\right)= & F_{1}, F_{2}, \\
P\left(w_{4}\right)= & F_{2}, \\
P\left(w_{5}\right)= & F_{1}, F_{3} .
\end{aligned}
$$

Notice that $P\left(F_{2}\right)$ is not substitutable and the two stable matchings

$$
\mu_{1}=\left(\begin{array}{ccc}
F_{1} & F_{2} & F_{3} \\
\left\{w_{1}, w_{2}\right\} & \left\{w_{3}, w_{4}\right\} & \left\{w_{5}\right\}
\end{array}\right)
$$

and

$$
\mu_{2}=\left(\begin{array}{cccc}
F_{1} & F_{2} & F_{3} & \varnothing \\
\left\{w_{3}, w_{5}\right\} & \left\{w_{1}, w_{2}\right\} & \varnothing & \left\{w_{4}\right\}
\end{array}\right)
$$

have the property that $F_{3}$ is single in $\mu_{2}$ but matched in $\mu_{1}$. Any $\mathscr{P}$ containing $P$ would violate axiom (OO) since $\mu_{\mathscr{W}}$ does not exist and $\mu_{1}=\mu_{\mathscr{F}}$ is not the worst stable matching for $w_{4}$.

\section{REFERENCES}

1. D. Gale and L. Shapley, College admissions and the stability of marriage, Amer. Math. Monthly 69 (1962), 9-15.

2. D. Gale and M. Sotomayor, Some remarks on the stable matching problem, Discrete Applied Mathematics 11 (1985), 223-232.

3. D. Gale and M. Sotomayor, Ms. Machiavelli and stable matching problem, Amer. Math. Monthly 92 (1985), 261-268.

4. A. Kelso and V. Crawford, Job matching, coalition formation, and gross substitutes, Econometrica 50 (1982), 1483-1504.

5. A. Roth, The evolution of the labor market for medical interns and residents: A case study in game theory, J. Pol. Econ. 92 (1984), 991-1016.

6. A. Roth, Conflict and coincidence of interest in job matching: Some new results and open questions, Math. Oper. Res. 10 (1985), 379-389. 
7. A. Roth, On the allocation of residents to rural hospitals: A general property of two-sided matching markets, Econometrica 54 (1986), 425-427.

8. A. Roth and M. Sotomayor, The college admissions problem revisited, Econometrica 57 (1989), 559-570.

9. A. Roth and M. Sotomayor, "Two-sided Matching: A Study in Game-Theoretic Modeling and Analysis," Econometrica Society Monographs, Vol. 18, Cambridge Univ. Press, Cambridge, U.K., 1990.

10. T. Sönmez, Strategy-proofness in many-to-one matching problems, Economic Design 1 (1996), 365-380. 